\title{
Evaluation of the SureX HPV genotyping test for the detection of high-risk HPV in cervical cancer screening
}

\author{
Baojun Wei ${ }^{1}$, Ping $\mathrm{Mei}^{2}$, Shengkai Huang ${ }^{1}$, Xueting $\mathrm{Yu}^{1}$, Tong Zhi ${ }^{3}$, Guojing Wang ${ }^{1}$, Xiaotian Xu ${ }^{1}$, Lin Xiao ${ }^{1}$, \\ Xin Dong ${ }^{1}$ and Wei Cui ${ }^{1 *}$ (D)
}

\begin{abstract}
Background: The SureX HPV genotyping test (SureX HPV test), which targets the human papillomavirus (HPV) E6/ E7 genes was compared with the Cobas 4800 and Venus HPV tests for detecting 14 high-risk HPV (HR-HPV) types in clinical referral and follow-up patients to evaluate its value for cervical cancer screening.

Methods: Two different populations were enrolled in the study. The first population comprised 185 cases and was used for comparing the SureX HPV test (Health, China) with the Cobas 4800 test (Roche, USA). The second population comprised 290 cases and was used for comparing the SureX HPV test (Health, China) with the Venus HPV test (Zhijiang, China). Polymerase chain reaction (PCR) sequencing was performed for further confirmation of discordant results.

Results: In the first population, the overall agreement rate was 95.6\% for 14 high-risk HPV types. Eight discordant cases were confirmed by PCR sequencing, which showed that the agreement rates were $75.0 \%$ between the SureX HPV test and PCR sequencing and $25.0 \%$ between the Cobas 4800 test and PCR sequencing $(P<0.01)$. In the second population, the overall agreement rate was $95.5 \%$. Thirteen discordant cases were confirmed by PCR sequencing, which showed that the agreement rates were $76.9 \%$ between the SureX HPV test and PCR sequencing and $23.1 \%$ between the Venus HPV test and PCR sequencing $(P<0.01)$. With cervical intraepithelial neoplasia grade $2+(C I N 2+)$ as the reference standard, the sensitivity values of the SureX HPV test and the Venus HPV test were $93.5 \%$ and $92.0 \%$, $(P>0.05)$, while the specificity values were $43.3 \%$ and $46.7 \%$, respectively $(P>0.05)$.
\end{abstract}

Conclusion: The SureX HPV test had good consistency with both the Cobas 4800 and Venus HPV tests for 14 HR-HPV types. In addition, it avoided some false negatives and false positives. Therefore, the SureX HPV test can be used for cervical cancer screening.

Keywords: High-risk human papillomavirus, HPV DNA test, Cervical cancer screening

*Correspondence: wendycuiwei@sina.cn

${ }^{1}$ Department of Clinical Laboratory, National Cancer Center/Cancer Hospital, Chinese Academy of Medical Sciences and Peking Union

Medical College, No. 17 Panjiayuannanli, Beijing 100021, People's Republic of China

Full list of author information is available at the end of the article

\section{Background}

Cervical cancer is one of the most common malignant tumors of the reproductive system in women. There were approximately 570,000 new cases of cervical cancer and 311,000 deaths worldwide in 2018 [1]. Persistent infection with high-risk human papillomavirus (HR-HPV) is recognized as the main cause of cervical cancer and precancerous lesions [2, 3]. HR-HPV DNA test for cervical specimens has been demonstrated as an effective original author(s) and the source, provide a link to the Creative Commons licence, and indicate if changes were made. The images or other third party material in this article are included in the article's Creative Commons licence, unless indicated otherwise in a credit line to the material. If material is not included in the article's Creative Commons licence and your intended use is not permitted by statutory regulation or exceeds the permitted use, you will need to obtain permission directly from the copyright holder. To view a copy of this licence, visit http://creativecommons.org/licenses/by/4.0/. The Creative Commons Public Domain Dedication waiver (http://creativeco mmons.org/publicdomain/zero/1.0/) applies to the data made available in this article, unless otherwise stated in a credit line to the data. 
approach to screen for cervical cancer and precancer, and has dramatically improved over the conventional cytology-based Pap smear [4]. Largely HR-HPV DNA test with polymerase chain reaction (PCR) is to detect the conserved region in L1 genes which code for the L1 capsid protein [5]. There is often an integration of HPV in the genome during progression from a low grade cervical lesion to cancer. And integration L1 expression can be lost. Therefore, this detection method for L1gene still needs improvement. But E6/E7 genes which encode oncogenic products remains always present, consequently, E6/E7 are pivotal in the development of cancer [6]. However, the degree of homology in this region limits digestion the detection of all HPV types [7]. Some studies have found that the detection of HPV E6/E7 DNA may be more accurate than the current DNA detection methods $[8,9]$. The SureX HPV genotyping test (SureX HPV test) is a novel HPV DNA detection method using capillary electrophoresis fragment analysis technology to target the HPV E6/E7 genes. In the present study, the SureX HPV test was compared with the Cobas 4800 test [10] and the Venus HPV test for detecting 14 types of HRHPV $[11,12]$ in clinical referral and follow-up patients to evaluate its value for cervical cancer screening.

\section{Materials and methods Study population}

Two different populations were enrolled in this study. The first population comprised 185 cases with cervical lesions, which contained detectable HPV DNA and underwent cervical cytopathological evaluation in the Department of Pathology of Guangdong Provincial People's Hospital between November 2017 and March 2018. The second population comprised 290 cases with cervical lesions, which contained detectable HPV DNA and underwent cervical histopathological evaluation in the Department of Clinical Laboratory of the National Cancer Center/Cancer Hospital between March 2018 and October 2018. The inclusion criteria included an age between 21 and 80 years, the absence of pregnancy, an intact cervix, no history of cervical lesions, and no history of chemotherapy, radiotherapy or surgical treatment.

\section{DNA extraction from cervical cells}

In this study, for each patient, flushed the female genital tract with normal saline, probed a specimen collection brush into the cervix, turned the brush to take the cervical secretions after $5 \mathrm{~s}$, and placed the brush in a $2 \mathrm{ml}$ volume collection tube. The cervical specimens collection were performed by an experienced doctor as detailed for each test. And the cervical specimens were stored in a refrigerator $\left(4^{\circ} \mathrm{C}\right)$ and analyzed within 14 days.
For the SureX HPV test, total cellular DNA was extracted from cervical specimens using the extraction work station Smart LabAssist-16/32 (Taiwan Advanced Nanotech Inc., Taiwan) according to the manufacturer's instructions. For Cobas 4800 HPV test, HPV DNA was extracted from cervical specimens using the automatic nucleic acid extractor cobas $\times 480$ DNA extractor (Roche Molecular Systems, Inc., USA). For the Venus HPV test, HPV DNA was extracted from cervical specimens using the automatic nucleic acid extractor Autrax workstation (Shanghai ZJ Bio-Tech Co., Shanghai, China). For the detection of 14 HR-HPV types, the three different method were used in accordance with the manufacturer's instructions. Negative and positive controls provided in the kits were included in each PCR test.

\section{SureX HPV genotyping test}

The SureX HPV test (Health Gene Technologies, Ningbo, China) utilized amplification of target HPV DNA by multiplex polymerase chain reaction (PCR) and capillary electrophoresis to detect and genotype 25 HPV types according to the length of specific amplification fragments. The HPV types were identified by the test including HPV $6,11,16,18,26,31,33,35,39,42,43,44,45,51$, $52,53,56,58,59,66,68,73,81,82$ and 83. For the SureX HPV test, specifically designed primers were targeted on early genes E6, E7 and E1 of HPV types, plasmid pcDNA $3.1(+)$ (pcDNA for short) and human $\beta$-globin locused to make sure the length of the amplified PCR products were at least 3 nucleotides difference. So, via PCR amplification of target DNA, 27 targets could be identified by capillary electrophoresis in a single analysis according to the length of PCR products. The measure of $\beta$-globin served as a quality control mechanism to confirm that a negative result was not due to inappropriate sample collection or failure of DNA extraction. The internal control pcDNA could monitor the PCR process and ensured that the testing procedure had been properly performed. Therefore, a validated specimen should show the specific peaks of pcDNA and $\beta$-globin. The peak height of pcDNA ought to be equal to or greater than $500 \mathrm{RFU}(\geq 500)$. When the peak of $\beta$-globin was absent indicating insufficient cervical cells, we suggested resampling should be conducted. For PCR amplification products $(1 \mu \mathrm{l})$ subjected to capillary electrophoresis in an ABI $3500 \mathrm{Dx} / 3500 \mathrm{xL} \mathrm{Dx}$ genetic analyzer, the cutoff value for determining specimens of HPV positive was:i) Signal of a HPV type $\geq 300$ RFU; or ii) Peak area ratio (the ratio of the peak area of a HPV type to the peak area of $\mathrm{pcDNA}) \geq 0.2$.

\section{Cobas $4800 \mathrm{HPV}$ test}

The cobas 4800 HPV test (Roche Molecular Systems, Inc., USA) used primers to define a sequence of approximately 
200 nucleotides within the L1 region of the HPV genome, which specifically detected 14 high-risk types (16, 18, $31,33,35,39,45,51,52,56,58,59,66$, and 68). The cobas4800 test mainly included two processes. Firstly, HPV DNA was extracted through automated sample preparation, and then the HPV and $\beta$-globulin target DNA sequences were amplified by PCR primers. The amplified target DNA sequences were combined with their corresponding fluorescent probes. There were 4 types of fluorescent probes for detection, namely HPV16, HPV18, $\beta$-globulin and 12 other high-risk HPVs. The probes were labeled with different fluorescent dyes, and the subtype of HPV in the sample were determined by real-time monitoring of fluorescent signals. In addition, $\beta$-globin was used as an internal control (IC) to ensure a sufficient sample quantity for HPV DNA detection. If the cycle threshold (Ct) cutoff value for HPV16 was $\leq 40.5$, a sample was considered HPV-positive; if the Ct cutoff value was $>40.5$ and $\beta$-globin was effective, it was considered negative; otherwise, it was considered invalid. If the Ct cutoff value for HPV18 or any of the 12 other highrisk types was $\leq 40$, a positive result was determined; and if the Ct cutoff value was $>40$ and $\beta$-globin was effective, a negative result was determined; otherwise, the result was considered invalid.

\section{Venus HPV genotyping test}

The Venus HPV genotyping test (Zhijiang Bio-Tech Co., Shanghai, China) was based on real-time fluorescence PCR technology and contained a specific ready-to-use system for the detection of 1515 types of HR- HPV genotypes, including HPV 16, 18, 31,33,35,39,45,51,52,56,58,5 $9,66,68,82$. Detection of amplified HPV DNA fragments was performed in the fluorimetric channels FAM, HEX/ VIC/JOE, TEXAS RED/Cal Red 610 and CY5 with the fluorescent quencher BHQ1. Human minibrain homolog (MNBH) was amplified as an internal control (IC) to indicate the presence of sufficient nucleic acid from the human $\mathrm{MNBH}$ gene. The $\mathrm{Ct}$ value was calculated. If the $C t$ value was $\leq 38.0$, a sample was considered HPV-positive. If the $\mathrm{Ct}$ value of the IC was $\leq 32.0$, and "undetermined" or "no CT" was displayed in the other channels, the sample was determined to be HPV-negative. If the $\mathrm{Ct}$ value was 38.0-40.0, the reaction was repeated. If the $\mathrm{Ct}$ value remained in this range and the amplification curve was a typical S-shape, the sample was considered HPV-positive; if the amplification curve was not a typical $\mathrm{S}$-type, as the sample was considered HPV-negative.

\section{Sequencing}

PCR sequencing was performed for further confirmation of discordant results. Sequencing reactions was targeted on type-specific E6/E7 gene and performed using the
ABI PRISM BigDye Terminator V3.0 kit (Applied Biosystems) and analyzed in an ABI 3730 genetic analyzer (Applied Biosystems) at Sangon Biotech Co. (Shanghai). DNA sequences were then compared with the sequences of known HPV types using the Basic Local Alignment Search Tool from the National Center for Biotechnology Information website (https://www.ncbi.nlm.nih.gov/ BLAST).

\section{Histological diagnosis}

The cytopathological diagnosis was based on the nomenclature of the Bethesda system of cervical cytology. The histopathological diagnosis was classified according to the WHO histological criteria for cervical tumors and was used as the gold standard, with cervical intraepithelial neoplasia grade 2 (CIN2) and higher (CIN2+) considered positive. Cytological diagnosis was performed by the Department of Pathology, Guangdong Provincial People's Hospital; pathological diagnosis, by the Cancer Hospital, Chinese Academy of Medical Sciences.

\section{Statistical analysis}

All statistical analyses were conducted using SPSS 23.0. The consistency checks were evaluated by the Kappa (k) values. Using CIN2+ as a reference, the sensitivity, specificity, and area under the receiver operating characteristic (ROC) curve (AUC) were calculated. All differences with $P$ values of $<0.05$ (two-tailed) were considered statistically significant.

\section{Results \\ Population 1 \\ HR-HPV infection}

The overall positive rates of the 185 cases for the SureX HPV test and the Cobas 4800 test were $72.4 \%(134 / 185)$ and $70.3 \%$ (130/185), respectively. Among the 185 cases, there were 12 NILM, 15 LSIL, 75 ASCUS, 43 HSIL+. In the different cervical cytopathological categories, no significant difference was observed in the positive rates of the 14 HR-HPV types between the SureX HPV test and the Cobas 4800 test $(P>0.05)$ (Table 1$)$.

\section{Agreement rate}

The results of the SureX HPV test and the Cobas 4800 test were shown in Table 2. The validation showed good agreement between the two different methods for the 14 HR-HPV types. The overall agreement rate was $95.6 \%$ $(177 / 185$, Kappa $=0.894)(95 \%$ confidence interval $[\mathrm{CI}]$ : $0.812-0.961)$.

There were 8 discordant results between the SureX HPV test and the Cobas 4800 tests; these results were confirmed by PCR sequencing, as shown in Table 3. Of the discordant results, 6 were positive by the SureX 
Table 1 Results of the SureX HPV and the Cobas $\mathbf{4 8 0 0}$ tests by cytological category

\begin{tabular}{|c|c|c|c|c|c|c|}
\hline & \multirow[t]{2}{*}{ Total (\%) } & \multicolumn{2}{|l|}{ SureX HPV } & \multicolumn{2}{|l|}{ Cobas 4800} & \multirow[t]{2}{*}{$P$ value } \\
\hline & & Positive (\%) & Negative (\%) & Positive (\%) & Negative (\%) & \\
\hline Total & 185 (100\%) & $134(72.4)$ & $51(27.6)$ & $130(70.3)$ & 55 (29.7) & 0.646 \\
\hline \multicolumn{7}{|c|}{ Cytological category } \\
\hline NILM & $12(6.5)$ & $4(33.3)$ & $8(66.7)$ & $4(33.3)$ & $8(66.7)$ & 1.00 \\
\hline LSIL & $55(29.7)$ & $48(87.3)$ & $7(12.7)$ & $47(85.5)$ & $8(14.5)$ & 0.781 \\
\hline ASCUS & $75(40.5)$ & $42(56.0)$ & $33(44.0)$ & $41(54.7)$ & $34(45.3)$ & 0.87 \\
\hline${ }^{\mathrm{a} H S I L+}$ & $43(23.2)$ & $40(93.0)$ & $3(7.0)$ & $38(88.4)$ & $5(11.6)$ & 0.458 \\
\hline
\end{tabular}

NILM negative for intraepithelial lesion or malignancy, LSIL low-grade squamous intraepithelial lesion, ASCUS atypical squamous cells of undetermined significance, HSIL high-grade squamous intraepithelial lesion, ACSH high atypical squamous cells, AGC atypical glandular cell, AIS adenocarcinoma in situ, SCC squamous cell carcinoma

${ }^{a}$ HSIL+ includes HSIL, ACSH, AGC, AIS, and SCC

Table 2 HR-HPV distribution of SureX HPV and Cobas 4800 results

\begin{tabular}{|c|c|c|c|c|c|c|c|c|c|}
\hline HPV genotype & SureX HPV & Cobas 4800 & $\begin{array}{l}{ }^{\mathrm{a}} \text { SureX+l } \\
{ }^{\mathrm{a}} \text { Cobas+ }\end{array}$ & $\begin{array}{l}\text { SureX+I } \\
\text { Cobas- }\end{array}$ & $\begin{array}{l}\text { SureX-I } \\
\text { Cobas+ }\end{array}$ & $\begin{array}{l}\text { SureX-I } \\
\text { Cobas- }\end{array}$ & $\begin{array}{l}\text { Positive } \\
\text { agreement } \\
\text { (\%) }\end{array}$ & Kappar & $95 \% \mathrm{Cl}$ \\
\hline HPV16 & 33 & 38 & 33 & 0 & 5 & 147 & 86.84 & 0.913 & $0.832-0.982$ \\
\hline HPV18 & 18 & 18 & 17 & 1 & 1 & 167 & 89.47 & 0.938 & $0.823-1.00$ \\
\hline Other & 100 & 98 & 95 & 5 & 3 & 85 & 92.23 & 0.915 & $0.851-0.968$ \\
\hline
\end{tabular}

a SureX, SureX HPV test; Cobas, Cobas 4800 test

Single HPV subtypes and multiple HPV subtypes were calculated together

Table 3 Discordance between the SureX HPV test and Cobas 4800 test

\begin{tabular}{lllll}
\hline No. & SureX HPV & Cobas $\mathbf{4 8 0 0}$ & PCR sequencing & Cytology \\
\hline 1 & 16 & Neg & 16 & LSIL \\
2 & Neg & 16 & 16 & ASCUS \\
3 & other $^{\text {a }}$ & Neg & other & ASCUS \\
4 & other & Neg & other & ASCUS \\
5 & other & Neg & other & ASC-H \\
6 & other & Neg & other & HSIL \\
7 & other & Neg & other & ASCUS \\
8 & Neg & other & other & ASCUS \\
\hline
\end{tabular}

Neg negative

a 12 HR-HPV is denoted "other" for Cobas 4800 test results

HPV test and PCR sequencing but negative by the Cobas 4800 test. The agreement rate was $75.0 \%(6 / 8)$ between SureX HPV test and PCR sequencing. Two cases were positive by the Cobas 4800 test and PCR sequencing but negative by the SureX HPV test. The agreement rate was $25.0 \%(2 / 8)$ between the Cobas 4800 test and PCR sequencing. The agreement rates were significantly different $(P<0.01)$.

\section{Population 2}

\section{HR-HPV infection}

Among the 290 cases, there were 33 diagnosed with normal cervix (11.4\%), 56 CIN1(19.3\%), 28 CIN2 (9.7\%), 35 CIN3 (12.1\%) and 138 cancer (47.6\%), and the percentage of CIN2 was $30.7 \%$ and CIN 2+ was $69.3 \%$.The overall positive rates of the 290 cases for the SureX HPV test and Venus HPV test were the same (78.3\%, 227/290), as shown in Table 4. The positive rates of the $14 \mathrm{HR}-\mathrm{HPV}$ types did not differ significantly between the two methods in the different histopathological categories $(P>0.05)$.

\section{Agreement rate}

The results of the SureX HPV test and the Venus HPV test were shown in Table 5. For both methods, HPV16 was the most frequently detected type, followed by HPV18 and HPV58 and HPV52. The concordance of the two methods for HPV 16, 18, 58, 52, 33, 51, 66, 35, 59, 56, and 39 was good, but was poor for HPV 45, 31, and 68 . The overall agreement rate was $95.5 \%(277 / 290$, Kappa $=0.838,95 \%$ CI: $0.750-0.906, P<0.01)$.

Thirteen results were discordant between the SureX HPV test and Venus HPV test; these results were confirmed by PCR sequencing, as shown in Table 6. Of the discordant results, 7 were positive by the SureX HPV 
Table 4 Results of the SureX HPV test and Venus HPV test by histopathological category

\begin{tabular}{|c|c|c|c|c|c|c|}
\hline & \multirow[t]{2}{*}{ Total (\%) } & \multicolumn{2}{|l|}{ SureX HPV } & \multicolumn{2}{|l|}{ Venus HPV } & \multirow[t]{2}{*}{$P$ value } \\
\hline & & Positive (\%) & Negative (\%) & Positive (\%) & Negative (\%) & \\
\hline Total & $290(100 \%)$ & $227(78.3)$ & $63(21.7)$ & $227(78.3)$ & $63(21.7)$ & 1.00 \\
\hline \multicolumn{7}{|c|}{ Histological category } \\
\hline Normal & $33(11.4)$ & $11(33.3)$ & $22(66.7)$ & $12(36.4)$ & $21(63.6)$ & 0.796 \\
\hline CIN1 & $56(19.3)$ & $28(50.0)$ & $28(50.0)$ & $30(53.6)$ & $26(46.4)$ & 0.705 \\
\hline CIN2 & $28(9.7)$ & 25 (89.3) & $3(10.7)$ & 25 (89.3) & $3(10.7)$ & 1.00 \\
\hline CIN3 & $35(12.1)$ & $34(97.1)$ & $1(2.9)$ & $30(85.7)$ & $5(14.3)$ & 0.088 \\
\hline$C A^{a}$ & $138(47.6)$ & $130(94.2)$ & $8(5.8)$ & $130(94.2)$ & $8(5.8)$ & 1.00 \\
\hline
\end{tabular}

${ }^{a}$ CA includes adenocarcinoma in situ (ACIS), squamous cell carcinoma (SCC), adenocarcinoma (AC) and adenosquamous carcinoma (ASC)

Table 5 HR-HPV distribution of SureX HPV and Venus HPV test results

\begin{tabular}{|c|c|c|c|c|c|c|c|c|c|}
\hline HPV genotype & SureX HPV & Venus HPV & $\begin{array}{l}\text { asureX+/ } \\
\text { aVenus+ }\end{array}$ & $\begin{array}{l}\text { SureX+/ } \\
\text { Venus- }\end{array}$ & $\begin{array}{l}\text { SureX-/ } \\
\text { Venus+ }\end{array}$ & $\begin{array}{l}\text { SureX-I } \\
\text { Venus- }\end{array}$ & $\begin{array}{l}\text { Positive } \\
\text { agreement } \\
\text { (\%) }\end{array}$ & Kappar & $95 \% \mathrm{Cl}$ \\
\hline HPV 16 & 144 & 143 & 138 & 6 & 5 & 141 & 92.6 & 0.924 & $0.876-0.966$ \\
\hline HPV18 & 28 & 32 & 27 & 1 & 5 & 257 & 81.8 & 0.889 & $0.786-0.970$ \\
\hline HPV58 & 26 & 23 & 22 & 4 & 1 & 263 & 81.5 & 0.889 & $0.772-0.978$ \\
\hline HPV52 & 18 & 20 & 17 & 1 & 3 & 269 & 81 & 0.887 & $0.758-0.976$ \\
\hline HPV33 & 14 & 13 & 13 & 1 & 0 & 276 & 92.9 & 0.916 & $0.863-1.00$ \\
\hline HPV51 & 10 & 9 & 9 & 1 & 0 & 280 & 90 & 0.946 & $0.775-1.00$ \\
\hline HPV66 & 8 & 8 & 7 & 1 & 1 & 281 & 77.8 & 0.871 & $0.594-1.00$ \\
\hline HPV35 & 4 & 3 & 3 & 1 & 0 & 286 & 75 & 0.855 & $0.329-1.00$ \\
\hline HPV59 & 6 & 4 & 4 & 2 & 0 & 284 & 66.7 & 0.797 & $0.329-1.00$ \\
\hline HPV56 & 7 & 8 & 6 & 1 & 2 & 281 & 66.7 & 0.795 & $0.491-1.00$ \\
\hline HPV39 & 10 & 13 & 9 & 1 & 4 & 276 & 64.3 & 0.696 & $0.502-0.864$ \\
\hline HPV45 & 5 & 3 & 2 & 3 & 1 & 284 & 33.3 & 0.493 & -0.862 \\
\hline HPV31 & 6 & 7 & 3 & 3 & 4 & 280 & 30 & 0.449 & -0.777 \\
\hline HPV68 & 4 & 3 & 0 & 4 & 3 & 283 & 0 & -0.012 & -0.025 \\
\hline
\end{tabular}

a SureX: SureX HPV test; Venus:Venus HPV test

Single HPV subtypes and multiple HPV subtypes were calculated together

test and PCR sequencing but negative by the Venus HPV test, and 3 were negative by the SureX HPV test and PCR sequencing but positive by the Venus HPV test. Thus, the agreement rate was $76.9 \%(10 / 13)$ between the SureX HPV test and PCR sequencing. Three cases were positive by the Venus HPV test and PCR sequencing but negative by the SureX HPV test. The agreement rate between the Venus HPV test and PCR sequencing was $23.1 \%(3 / 13)$. The agreement rates differed significantly $(P<0.01)$.

\section{Sensitivity and specificity}

With CIN2+ as the reference standard, receiver operating characteristic (ROC) curve analysis was used to calculate the sensitivity and specificity. The sensitivity values of the SureX HPV test and Venus HPV test were 93.5\% and $92.0 \%,(P>0.05)$, and the specificity values were $43.3 \%$ and $46.7 \%$, respectively $(P>0.05)$. The AUCs for the SureX HPV test and Venus HPV test were 0.751 (95\% CI: 0.683-0.819, $P<0.01$ ) and 0.727 (95\% CI: 0.658-0.96, $P<0.01$ ), respectively (Fig. 1 ).

\section{Discussion}

In the detection of HPV DNA PCR amplification, the selection of the target region and the design of the primers are particularly important for maximizing the amplification efficiency [13]. Because of the high conservation of HPV L1 DNA across genotypes, universal primers can be designed to amplify DNA from multiple genotypes; L1 DNA from different genotypes presents sufficient sequence differences to allow further analysis of specific 
Table 6 Discordance between the SureX HPV test and Venus HPV test

\begin{tabular}{lllll}
\hline No. & SureX HPV & Venus HPV & Sequencing & Histology \\
\hline 1 & 16 & Neg & 16 & CIN3 \\
2 & 16 & Neg & 16 & CIN3 \\
3 & 16 & Neg & 16 & SCC \\
4 & other $^{\text {a }}$ & Neg & other & SCC \\
5 & other & Neg & other & SCC \\
6 & other & Neg & other & CIN3 \\
7 & other & Neg & other & CIN2 \\
8 & Neg & 16 & 16 & AC \\
9 & Neg & 18 & 18 & SCC \\
10 & Neg & $16 /$ other & $16 /$ other & SCC \\
11 & Neg & 16 & Neg & CIN1 \\
12 & Neg & other & Neg & SCC \\
13 & Neg & other & Neg & CIN2 \\
\hline
\end{tabular}

Neg negative

a 12 HR-HPV, including HPV $31,33,35,39,45,51,52,56,58,59,66$, and 68 , is denoted as "other"

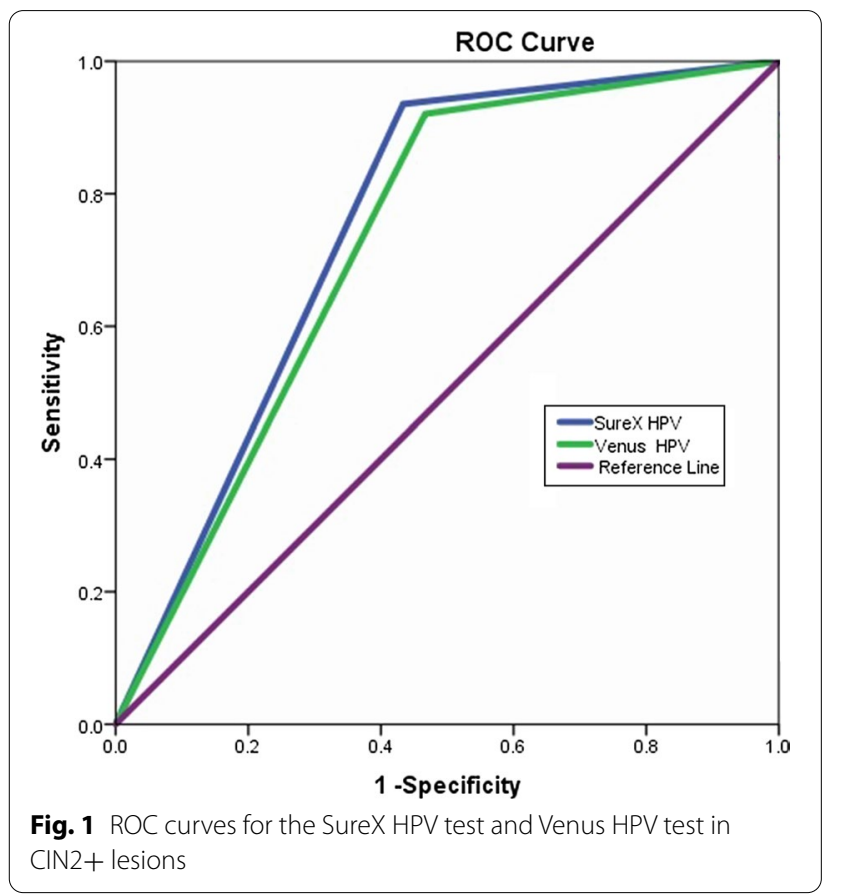

genotypes by other methods [14]. Therefore, most HPV DNA tests currently on the market detect HPV L1 DNA [15]. The Cobas 4800 HR-HPV test uses HPV L1 DNA as an amplification target and can detect HPV16, HPV18, and 12 other HR-HPV types. The Venus HPV genotyping test also uses HPV L1 DNA as the detection target and can detect 15 HR-HPV subtypes. The Venus HPV test is widely used in China because of its specificity and sensitivity, which are better than those of similar products, and because it is easy to perform and inexpensive.

However, HPV L1 may be lost during the integration of HPV DNA into the host genome, and HPV tests based on L1 may lead to missed diagnoses of cervical cancer, which may affect the clinical sensitivity and positive predictive value of such tests $[16,17]$. Research has shown that $0.3 \%$ of CIN2 and 3.94\% of CIN3 lesions among HPV16positive women were L1-negative [18]. Some researchers think that detection methods based on the E6/E7 gene are better than those based on the L1 gene [19, 20].

In this study, we compared the SureX HPV test with the Cobas 4800 and Venus HPV tests, which are widely used at home and abroad. The overall agreement rates were $95.3 \%(162 / 170$, Kappa $=0.894)$ between the SureX HPV and the Cobas 4800 tests, and were 94.5\% (274/290, Kappa $=0.838$ ) between the SureX HPV and the Venus HPV tests. Therefore, good concordance was shown for detecting the 14 HR-HPV types between the SureX HPV and the Cobas 4800 tests and between the SureX HPV and the Venus HPV tests.

Persistent HR-HPV infection is the key factor for cervical cancer. The most common HPV genotypes causing cervical cancer are HPV 16 and 18 [21, 22]. The results of a large-scale multicenter epidemiological study in China showed that the most common types of HPV causing infection were HPV16 and HPV18, followed by HPV52 and HPV58 [23]. In this study, the results in the second population were consistent with those of that large-scale study.

To determine the actual HPV infection and show the accuracy of the SureX HPV test, PCR sequencing targeted on type-specific E6/E7 gene was performed to further confirm discordant results. In our study, 6 cases were positive by the SureX HPV test and PCR sequencing but negative by the Cobas 4800 test, and 7 were positive by the SureX HPV test and PCR sequencing but negative by the Venus HPV test. As both the Cobas 4800 and the Venus HPV tests target HPV L1 DNA, these 13 results may be false negatives due to missed detection of HPV L1 DNA. In addition, 2 cases were positive by the Cobas 4800 test and PCR sequencing, while 3 cases were negative by the SureX HPV test, and 3 cases were positive by the Venus HPV test and PCR sequencing but negative by the SureX HPV test. The reason for this discrepancy may be that the results of capillary electrophoresis in the SureX HPV test showed a peak height of pcDNA in the five samples of approximately $500 \mathrm{RFU}$, which may have resulted in low amplification efficiency because of low levels of HPV DNA in the cervical cell specimens. Although HPV DNA was extracted by three different automatic nucleic acid extractor in our study, the three automatic nucleic acid extractor were all magnetic bead 
methods and the three test had an internal control (IC) to indicate the presence of sufficient nucleic acid.

Additionally, studies had shown that although the HRHPV DNA test had high sensitivity, the potential hazard of this method was that it could detect a large number of women with false-positive results, who were likely to have transient infections. After a few months, HPV is cleared naturally by the body without causing cervical cancer or precancerous lesions [24]. Three patients in our study were positive by the Venus HPV test but negative by the SureX HPV test and sequencing; these results may be false positives. The results of this study indicated that the agreement rate between the SureX HPV test and PCR sequencing were $75.0 \%(6 / 8)$ in the first population and $76.9 \%(10 / 13)$ in the second population; therefore, the SureX HPV test could be used for HR-HPV detection.

The method of screening cervical cancer and precancerous lesions by detecting HPV DNA was characterized by high sensitivity, while specificity was related to the positive rate of HPV. The specificity differed greatly among different populations [25]. With CIN2+ as the reference standard, the sensitivity values of the SureX HPV test (93.5\%) was higher than the Venus HPV test (92.0\%), and the specificity values of the SureX HPV test (43.3\%) was lower than that of the Venus HPV test (46.7\%). Some studies showed that the specificity of HPV DNA detection was lower than $50 \%[25,26]$. The AUC of the SureX HPV test were 0.751 (95\% CI:0.683- 0.819, $P<0.01$ ) and the Venus HPV test were 0.727 (95\% CI: 0.658-0.96, $P<0.01)$. Therefore, there was reasonable consistency between the SureX HPV test and Venus HPV test.

In the study, there were 8 cases was negative to three different HR-HPV test and PCR sequence in HSIL+ or CIN2+ population. The possible reasons may be inappropriate sample collection, or the low copies of HPV DNA in the sample, or the patient factors, such as the patient had been treated but not informed.

Compared with previously developed and wisely used HPV detection methods such as real time PCR, the SureX HPV test described PCR-capillary electrophoresis method could achieve detection and identification of $25 \mathrm{HPV}$ genotypes in one tube with targeting on specific oncogenic E6/E7. Normally it took around $6 \mathrm{~h}$ to have 96 specimens detected and reported if the capillary electrophoresis platform was 24-channel equipped. The testing cost is competitive with regular test methods.

\section{Conclusion}

In summary, in this study, we compared a novel HPV genotyping test, the SureX HPV test, with the Cobas 4800 and the Venus HPV tests. The SureX HPV test had good consistency with the Cobas 4800 and the Venus HPV tests for detecting 14 HR-HPV types. In addition, the SureX HPV test could avoid some false-negative and false-positive results, and its sensitivity and specificity for pathological grade CIN2+ lesions was equivalent to that of the Venus HPV test. Therefore, the SureX HPV genotyping test is a novel method detecting HPV DNA, which utilizes PCR amplification and capillary electrophoresis to identify $25 \mathrm{HPV}$ types in a single analysis, and it is an accurate, safe, and inexpensive HPV detection method. However, as the population selected for this study was the primary screening-positive population rather than the general population, further comparative analysis of these three methods through large-sample studies in the general population to provide a basis for the development of a large-scale cervical cancer screening strategy.

\section{Abbreviations \\ AGC: Atypical glandular cell; AIS: Adenocarcinoma in situ; ASCUS: Atypical squamous cells of undetermined significance; $\mathrm{ACSH}$ : High atypical squamous cells; CA: Cancer; Cl: Confidence interval; CIN: Cervical intraepithelial neoplasia; Ct: Cycle threshold; HR-HPV: High-risk human papillomavirus; HSIL: High-grade squamous intraepithelial lesion; IC: Internal control; LSIL: Low-grade squamous intraepithelial lesion; MNBH: Human minibrain homolog; NILM: Negative for intraepithelial lesion or malignancy; Neg: Negative; PCR: Polymerase chain reaction; ROC: Receiver operating characteristic curve (AUC); RFU: Relative fluorescence units; SCC: Squamous cell carcinoma; SureX HPV test: SureX HPV genotyping test}

\section{Acknowledgements}

We acknowledge support from the National Natural Science Foundation of China, the CAMS Innovation Fund for Medical Sciences (CIFMS) and the Beijing Hope Run Special Fund of Cancer Foundation of China.

\section{Authors' contributions}

B.W. carried out the experiments and prepared the manuscript; P.M. and X.Y. collected the samples and carried out the experiments; S.H. and supported the manuscript writing; T.Z. and G.W. carried out the control experiments; X.X., L.X. and X.D. analysed data; W.C. provided the original ideas and experimental structure and supported the experiments and manuscript writing. All authors read and approved the final manuscript.

\section{Funding}

This work was supported by the National Natural Science Foundation of China (Grant No.81772272), the CAMS Innovation Fund for Medical Sciences (CIFMS) (Grant No. 2017- 12M-3-005) and the Beijing Hope Run Special Fund of Cancer Foundation of China (LC2018B16).

Availability of data and materials

Not applicable. All relevant data are within the paper.

Ethics approval and consent to participate

The study was approved by the Ethics Committee at National Cancer Center/ Cancer Hospital.

\section{Consent for publication}

Not applicable.

\section{Competing interests}

The authors declare that they have no competing interests.

\section{Author details}

${ }^{1}$ Department of Clinical Laboratory, National Cancer Center/Cancer Hospital, Chinese Academy of Medical Sciences and Peking Union Medical College, No. 17 Panjiayuannanli, Beijing 100021, People's Republic of China. ${ }^{2}$ Department of Pathology, Guangdong Provincial People's Hospital, Guangdong Academy of Medical Sciences, Guangzhou, People's Republic of China. ${ }^{3}$ Department 
of Clinical Laboratory, Beijing Fengtai Youanmen Hospital, Beijing, People's Republic of China.

Received: 3 June 2020 Accepted: 22 September 2020

Published online: 09 November 2020

\section{References}

1. Bray F, Ferlay J, Soerjomataram I, Siegel RL, Torre LA, Jemal A. Global cancer statistics 2018: GLOBOCAN estimates of incidence and mortality worldwide for 36 cancers in 185 countries. CA Cancer J Clin. 2018:68:394-424.

2. Iarc WG. Human papillomaviruses. IARC Monogr Eval Carcinog Risks Hum. 2007:90:1-636

3. Chan CK, Aimagambetova G. Human papillomavirus infection and cervical cancer: epidemiology, screening, and vaccination-review of current perspectives. J Oncol. 2019;2019:3257939. https://doi. org/10.1155/2019/3257939.

4. Bosch FX. Human papillomavirus: science and technologies for the elimination of cervical cancer. Expert Opin Pharmacother. 2011;12:2189-204.

5. Buck CB, Day PM, Trus BL. The papillomavirus major capsid protein L1. Virology. 2013;445:169-74.

6. Tjalma WA, Depuydt CE. Cervical cancer screening: which HPV test should be used-L1 or E6/E7? Eur J Obstet Gynecol Reprod Biol. 2013;170:45-6.

7. Lungu O, Sun XW, Wright TC Jr, Ferenczy A, Richart RM, Silverstein S. A polymerase chain reaction-enzyme-linked immunosorbent assay method for detecting human papillomavirus in cervical carcinomas and high-grade cervical cancer precursors. Obstet Gynecol. 1995;85:337-42.

8. Walboomers JM, Jacobs MV, Manos MM, Bosch FX, Kummer JA, Shah KV, Snijders PJ, Peto J, Meijer CJ, Muñoz N. Human papillomavirus is a necessary cause of invasive cervical cancer worldwide. J Pathol. 1999;189:12-9.

9. Saslow D, Solomon D, Lawson HW, Killackey M, Kulasingam SL, Cain J, Garcia FA, Moriarty AT, Waxman AG, Wilbur DC, et al. American Cancer Society, American Society for Colposcopy and Cervical Pathology, and American Society for Clinical Pathology screening guidelines for the prevention and early detection of cervical cancer. CA Cancer J Clin. 2012;62:147-72

10. Pesic A, Krings A, Hempel M, Preyer R, Chatzistamatiou K, Agorastos T, Kaufmann AM. CIN2+ detection of the HPV DNA array genotyping assay in comparison with the Cobas 4800 HPV test and cytology. Virol J. 2019;16:92.

11. Burd EM. Human papillomavirus laboratory testing: the changing paradigm. Clin Microbiol Rev. 2016;29:291-319.

12. Muñoz N, Bosch FX, de Sanjosé S, Herrero R, Castellsagué X, Shah KV, Snijders PJ, Meijer CJ. Epidemiologic classification of human papillomavirus types associated with cervical cancer. N Engl J Med. 2003;348:518-27.

13. Buck CB, Cheng N, Thompson CD, Lowy DR, Steven AC, Schiller JT, Trus BL. Arrangement of L2 within the papillomavirus capsid. J Virol. 2008;82:5190-7.
14. Molijn A, Kleter B, Quint W, van Doorn LJ. Molecular diagnosis of human papillomavirus (HPV) infections. J Clin Virol. 2005;32(Suppl 1):S43-51.

15. Shen-Gunther J, Yu X. HPV molecular assays: defining analytical and clinical performance characteristics for cervical cytology specimens. Gynecol Oncol. 2011:123:263-71.

16. Lambert PF, Pan H, Pitot HC, Liem A, Jackson M, Griep AE. Epidermal cancer associated with expression of human papillomavirus type 16 E6 and E7 oncogenes in the skin of transgenic mice. Proc Natl Acad Sci USA. 1993:90:5583-7.

17. Morris BJ. Cervical human papillomavirus screening by PCR: advantages of targeting the E6/E7 region. Clin Chem Lab Med. 2005:43:1171-7.

18. Roberts CC, Tadesse AS, Sands J, Halvorsen T, Schofield TL, Dalen A, Skjeldestad FE, Jansen KU. Detection of HPV in Norwegian cervical biopsy specimens with type-specific PCR and reverse line blot assays. J Clin Virol. 2006;36:277-82.

19. de Freitas AC, Coimbra EC, Leitão Mda C. Molecular targets of HPV oncoproteins: potential biomarkers for cervical carcinogenesis. Biochim Biophys Acta. 2014;1845:91-103.

20. Moody CA, Laimins LA. Human papillomavirus oncoproteins: pathways to transformation. Nat Rev Cancer. 2010;10:550-60.

21. de Sanjose S, Quint WG, Alemany L, Geraets DT, Klaustermeier JE, Lloveras B, Tous S, Felix A, Bravo LE, Shin HR, et al. Human papillomavirus genotype attribution in invasive cervical cancer: a retrospective cross-sectional worldwide study. Lancet Oncol. 2010;11:1048-56.

22. Guan P, Howell-Jones R, Li N, Bruni L, de Sanjosé S, Franceschi S, Clifford GM. Human papillomavirus types in 115,789 HPV-positive women: a meta-analysis from cervical infection to cancer. Int J Cancer. 2012;131:2349-59.

23. Chen W, Zhang X, Molijn A, Jenkins D, Shi JF, Quint W, Schmidt JE, Wang P, Liu YL, Li LK, et al. Human papillomavirus type-distribution in cervical cancer in China: the importance of HPV 16 and 18. Cancer Causes Control. 2009:20:1705-13.

24. Steben $M$, Duarte-Franco E. Human papillomavirus infection: epidemiology and pathophysiology. Gynecol Oncol. 2007;107:S2-5.

25. Szarewski A, Mesher D, Cadman L, Austin J, Ashdown-Barr L, Ho L, Terry G, Liddle S, Young M, Stoler M, et al. Comparison of seven tests for highgrade cervical intraepithelial neoplasia in women with abnormal smears: the predictors 2 study. J Clin Microbiol. 2012;50:1867-73.

26. Nakamura M, Nakade K, Orisaka S, Iwadare J, Mizumoto Y, Fujiwara H. Comparison study of BD onclarity HPV with digene HC2 high-risk HPV DNA test and Roche Cobas 4800 HPV for detecting high-risk human papillomavirus in Japan. Am J Clin Pathol. 2019:151:263-9.

\section{Publisher's Note}

Springer Nature remains neutral with regard to jurisdictional claims in published maps and institutional affiliations.

Ready to submit your research? Choose BMC and benefit from

- fast, convenient online submission

- thorough peer review by experienced researchers in your field

- rapid publication on acceptance

- support for research data, including large and complex data types

- gold Open Access which fosters wider collaboration and increased citations

- maximum visibility for your research: over 100M website views per year

At BMC, research is always in progress.

Learn more biomedcentral.com/submissions 